\title{
Novel Microthermal Sensor for Quantification of Methanol in Water for Direct Methanol Fuel Cells
}

\author{
B. Schmitt, C. Kiefer, A. Schütze \\ Laboratory for Measurement Technology, Saarland University, Saarbrücken, Germany, \\ b.schmitt@LMT.uni-saarland.de
}

\begin{abstract}
A novel, simple sensor principle for determination of the ratio of fluids in a mixture is investigated. It makes use of the different thermal properties of the components in the mixture like thermal conductivity and heat capacity. Through their determination the ratio of components of binary mixtures can be measured if the components are known. When applying a heat pulse the temperature rise after a given time is used to determine the mixture ratio. Using microfabricated structures the sensor is capable of measuring the mixture ratio with an accuracy of approximately $2 \%$ in single shot experiments in less than a second. In Direct Methanol Fuel Cells the sensor can be used in a closed loop control of the methanol amount in deionized water to ensure the optimum working point.
\end{abstract}

Key words: Microthermal sensor, Direct Methanol Fuel Cell, fluid mixture ratio, low cost, and closed loop control of methanol concentration.

\section{Introduction}

The analysis of fluid mixtures with regard to their composition over a wide concentration range is still a major challenge. Such sensors are required, for example, for Direct Methanol Fuel Cells (DMFC). Here, methanol from the storage tank is dosed into a water flow and consumed in the DMFC. To achieve the optimum efficiency of the cell the methanol amount has to be kept constant at a molar concentration of approx. $0.3 \mathrm{M}$ to $1 \mathrm{M}$, depending on the type of the DMFC [1-5], corresponding to a volume concentration of approx. $1.2 \mathrm{vol} \%$ to $4.1 \mathrm{vol} \%$. Since the methanol is only partially consumed in the DMFC, the remaining mixture can be used again to feed the cell if the optimum methanol concentration is restored. For this purpose a sensor is required that allows a closed loop control keeping the methanol concentration at the optimum working point to achieve highest efficiency and long lifetime $[5,6]$, see Fig. 1. A measurement resolution of $0.1 \%$ of the methanol concentration would be ideal; however, as there are currently no sensors available for this application, even a resolution of $1 \%$ would be a major improvement. In addition to accurate and stable measurement, low cost and low power consumption of the sensor are required to address applications such as DMFC power supplies for laptops and similar equipment. Other possible applications include urea-water-mixtures used in Selective
Catalytic Reduction (SCR) systems for $\mathrm{NO}_{\mathrm{x}}$ reduction of exhaust gases, water content in oil for condition monitoring of e.g. hydraulic systems and also the detection of food contaminations.

The sensor principle under investigation is sensitive to the thermal properties of binary mixtures such as thermal conductivity and heat capacity and allows determination of their ratio based on the knowledge of the components in the mixture. Because of the simple construction of the microstructured sensor, and the uncomplicated signal analysis, the sensor principle is well-suited for low-cost applications. At the same time, it offers better long-term stability than chemical sensors by making use of physical properties of the measured liquid.

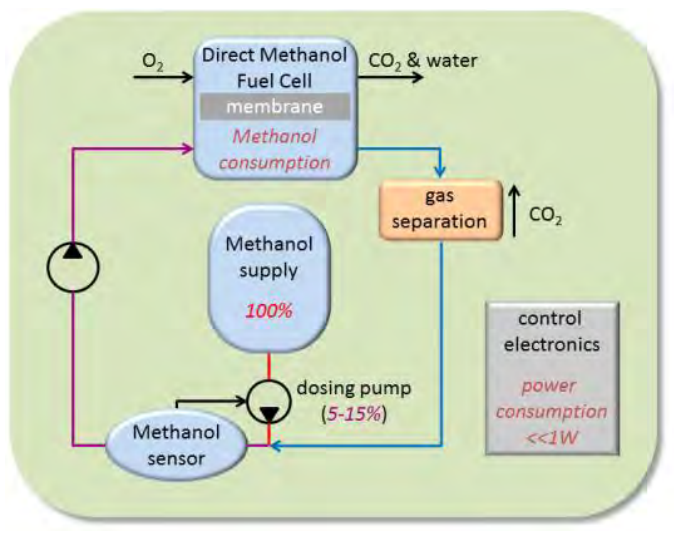

Fig. 1. Schematic of a DMFC-system with closed loop control of the methanol concentration. 


\section{Measurement principle}

For methanol in water, Fig. 2 shows the resulting thermal properties of the mixture at $20^{\circ} \mathrm{C}$, depending on the volume concentration of methanol. The thermal conductivity $\lambda$ decreases almost linearly from $0.59 \mathrm{~W} /(\mathrm{m} \cdot \mathrm{K})$ for pure water to $0.21 \mathrm{~W} /(\mathrm{m} \cdot \mathrm{K})$ for methanol [7]. On the other hand, the specific heat capacity $c_{p}$ of the mixture shows a nonlinear behavior with a non-unique relation in the range up to $20 \mathrm{vol} \%$, resulting in a maximum at approx. 12 vol\% [8].

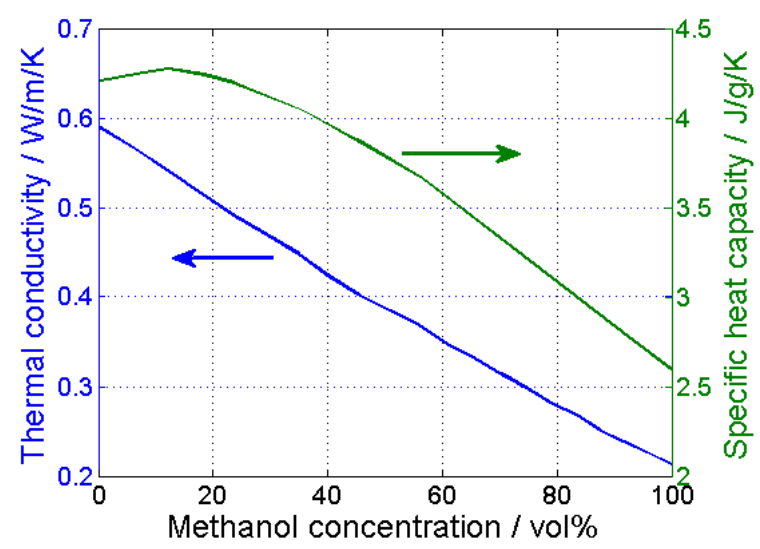

Fig. 2. Thermal conductivity and specific heat capacity of methanol/water-mixtures at $20^{\circ} \mathrm{C}[7,8]$.

By measuring one or both of these properties the mixture ratio of methanol in water can be determined. The measurement principle under investigation is based on the spatial and temporal spreading of an applied heat pulse, which is affected by both thermal properties of the mixture covering the sensor. The temperature increase of the heater itself but also of up to four temperature sensors arranged around the central heater as shown in Fig. 3 is measured. Varying distances between the temperature sensors and the heater and different heater diameters are tested as well as symmetric and asymmetric layouts to improve measurement accuracy and resolution. The active area of the sensor is currently approx.

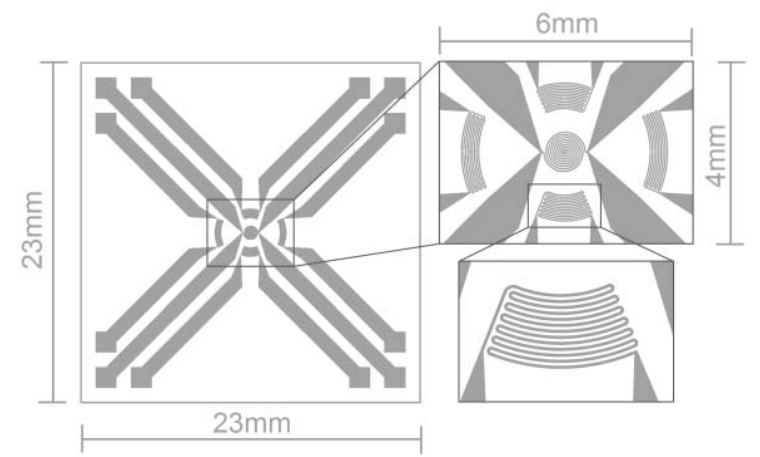

Fig. 3. Top view of the microstructured sensor layout with enlarged view of the central heater (approx. area 0.2-0.8 $\mathrm{mm}^{2}$ ) and the four surrounding temperature sensors.
$24 \mathrm{~mm}^{2}$, but can be reduced further in the future.

The resistance of the heater is measured with a four-terminal setup for accurate temperature determination. By evaluating the measured temperature increase the ratio of the components in the binary mixture can be determined. For a more robust evaluation of the sensor signal fitting of exponential curves to the temperature increase is also investigated. By determining the pre-exponential factor as well as the time constant which reflect both the thermal conductivity and the heat capacity it would be possible to also address the measurement of ternary mixtures. To take different contributions into account, the fitfunctions contain two exponential terms as shown in eq. (1).

$$
T(t)=c-a \cdot \exp \left(-\frac{t}{\tau_{1}}\right)-b \cdot \exp \left(-\frac{t}{\tau_{2}}\right)
$$

Here, $T(t)$ is the mean temperature of the central heater after the heating time $t, \tau_{1}$ and $\tau_{2}$ are the time constants of the temperature increase with $\tau_{1}<\tau_{2}, a$ and $b$ are the preexponential factors and the constant $c$ is the steady state value of the heater temperature that would be reached with a given constant heating power after infinite time.

\section{Measurement setup}

The sensor chip is integrated in a fluid chamber (Fig. 4) in which the heater and temperature sensors are contacted using contact pins. The inside chamber width can be changed using a screw and is set to $1 \mathrm{~mm}$. In higher chambers natural convection was observed in simulations of the measurement principle using Comsol Multiphysics (Comsol AB). Kapton HN (DuPont)

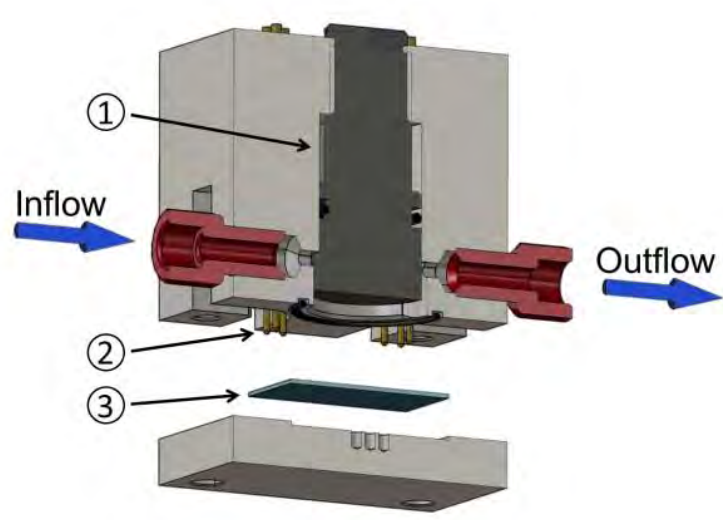

Fig. 4. Cross section of the measurement chamber with sensor chip (3) and arrows indicating the flow direction. The inside chamber width can be changed using the screw (1); the chip is contacted with contact pins (2). 
a polyimide foil with a thickness of $125 \mu \mathrm{m}$, is used as substrate for the sensor because of its very low thermal conductivity of $0.12 \mathrm{~W} / \mathrm{m} \cdot \mathrm{K}$ [9], ensuring that the heat is dissipated from the heater mainly into the fluid under test. The coefficient of thermal expansion in the temperature range of $30^{\circ} \mathrm{C}$ to $100^{\circ} \mathrm{C}$ is approx. $17 \mathrm{ppm} / \mathrm{K}$ and can induce stress if the material of the microstructured heater and sensors is not adapted. Aluminum thin films lithographically structured are used because of the high temperature coefficient of resistance (see Tab. 1). To increase the adhesion on the foil a $20 \mathrm{~nm}$ Titanium layer will be tested as adhesion promoter, whereat the possible stress in the layer due to the different thermal expansion coefficients has to be considered. Alternatively, the use of nickel with a higher temperature coefficient would lead to a better resolution and sensitivity of the sensor.

Tab. 1: Comparison of possible materials for heater and temperature sensors [10]

\begin{tabular}{|c|c|c|c|}
\hline Metal & $\begin{array}{c}\text { Temp. } \\
\text { coeff. of } \\
\text { resistance } \\
\text { at } 20^{\circ} \mathrm{C} / \\
1 / \mathrm{K}\end{array}$ & $\begin{array}{c}\text { Electrical } \\
\text { resistivity at } \\
20^{\circ} \mathrm{C} \\
/ \mathrm{Ohm} \cdot \mathrm{m}\end{array}$ & $\begin{array}{c}\text { Coefficient } \\
\text { of thermal } \\
\text { expansion } \\
\text { at } 25^{\circ} \mathrm{C} \\
/ \mathrm{ppm} / \mathrm{K}\end{array}$ \\
\hline $\mathrm{Al}$ & $4.5 \cdot 10^{-3}$ & $2.4 \cdot 10^{-8}$ & 23.1 \\
\hline $\mathrm{Cr}$ & $2.3 \cdot 10^{-3}$ & $11.8 \cdot 10^{-8}$ & 4.9 \\
\hline $\mathrm{Ni}$ & $5.6 \cdot 10^{-3}$ & $6.9 \cdot 10^{-8}$ & 13.4 \\
\hline $\mathrm{Pt}$ & $4.1 \cdot 10^{-3}$ & $10.5 \cdot 10^{-8}$ & 8.8 \\
\hline $\mathrm{Ti}$ & $2.8 \cdot 10^{-3}(1)$ & $39.0 \cdot 10^{-8}(2)$ & 8.6 \\
\hline
\end{tabular}

(1) measured value [11]; (2) at $0^{\circ} \mathrm{C}$

\section{Measurement results}

Fig. 5 shows the raw data of a series of measurements for varying amounts of methanol in deionized water in the range of $0 \mathrm{vol} \%$ to $20 \mathrm{vol} \%$. With increasing methanol concentration the power dissipated in the heater leads to a larger temperature increase as the thermal conductivity $\lambda$ of the mixture decreases. Three measurements for each concentration are shown indicating satisfactory repeatability. Determination of the mixture is possible based on the temperature increase of the heater after a given time, i.e. $0.5 \mathrm{~s}$, with an accuracy of approximately $2 \%$ in single shot experiments and higher for repeated temperature pulses. The applied heating power is approx. $60 \mathrm{~mW}$ for a heater with a diameter of $1 \mathrm{~mm}$. By using a smaller heater the heating power can be reduced without a loss of sensitivity. Fig. 6 shows the corresponding temperature increase after a certain measurement time.

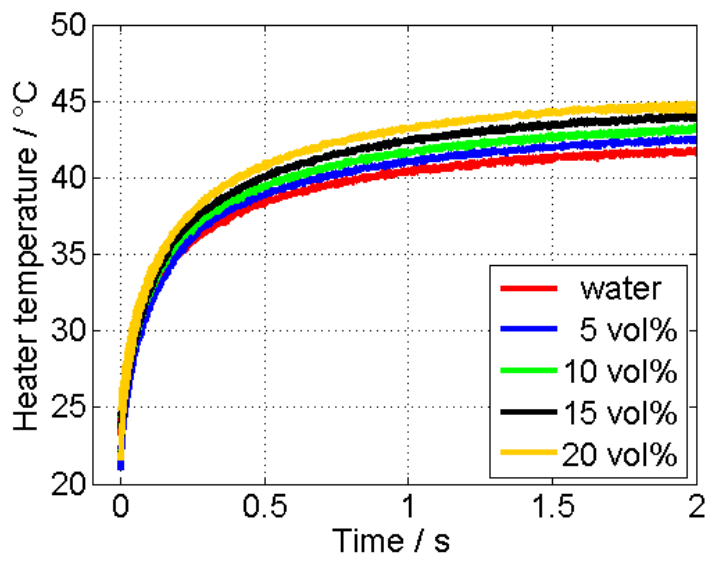

Fig. 5. Series of measurements of varying concentrations of methanol in deionized water.

The increase of the heater temperature shows a nearly linear dependence on the methanol concentration in the range typically used for Direct Methanol Fuel Cells. The used heating power results in temperature differences of $0.12,0.14$ and $0.15 \mathrm{~K} / \mathrm{vol} \%$ after $0.5,1$ and 2 seconds, respectively. Therefore, to reduce the heating power the duration of the heat pulse can be shortened without a significant loss of accuracy.

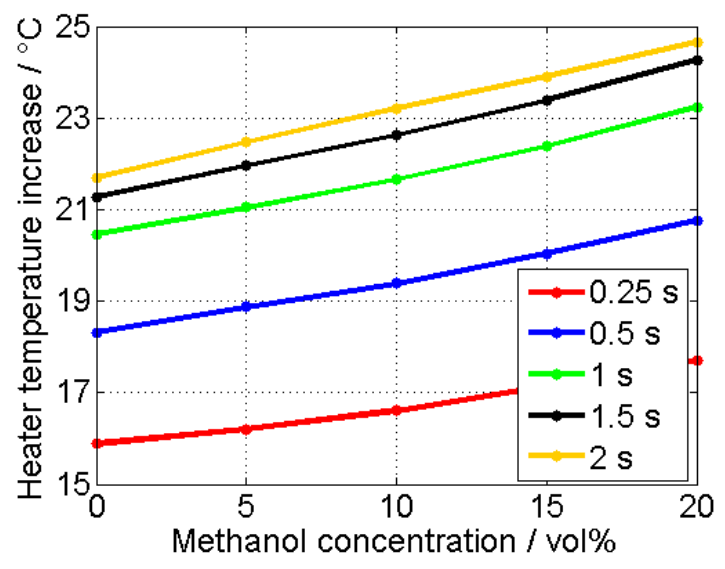

Fig. 6. Temperature increase after a certain measurement time for measurements of different methanol concentrations in deionized water.

Increasing heating power also almost linearly increases the temperature difference and the resolution of the measurements, but the boiling point of methanol/water-solutions is reduced with increasing methanol amount reaching a value of only $88.3^{\circ} \mathrm{C}$ for a concentration of $20 \mathrm{vol} \%$ [12]. It also has to be considered that the measured heater temperature is the averaged value while in the middle of the heater the temperature is higher. This difference can reach values of up to $20^{\circ} \mathrm{C}$ according to finite element simulations. To prevent the mixture from boiling, the heating power is reduced limiting the maximum average temperature to $50^{\circ} \mathrm{C}$. 
In addition to the measurements shown in Fig. 5, the sensor was tested over a larger concentration range up to $80 \mathrm{vol} \%$ methanol in water. The results of three measurements at each concentration were fitted with eq. (1) using MATLAB (The MathWorks, Inc.), the resulting parameters are shown in Figs. 7 and 8.

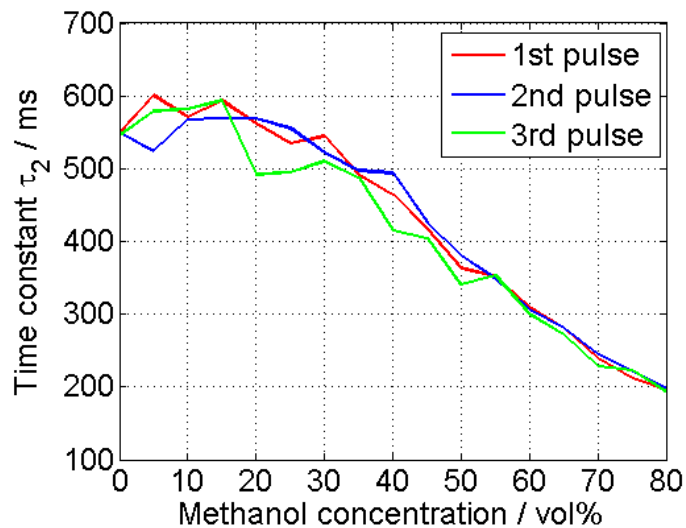

Fig. 7. Time constant $\tau_{2}$ of the fitted temperature increase of the heater for three heat pulses showing typical variations in the measurement.

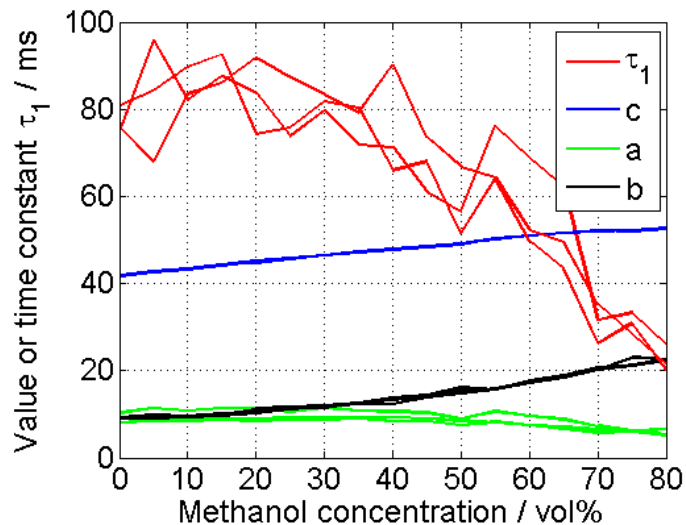

Fig. 8. Time constant $\tau_{1}$, pre-exponential factors $a$ and $b$ and constant $c$ of the fitted temperature increase of the heater.

Both time constants show a similar trend reflecting the change of the mixture properties. Especially $\tau_{2}$ shows a dependence very similar to the heat capacity of the mixture (cf. Fig. 2).

If the system could be modeled with only a single time constant $\tau$ (i.e. as a thermal RC circuit) this would be determined by the thermal properties according to eq. (2).

$\tau=\alpha \cdot \frac{c_{p}}{\lambda}$

Here, $\alpha$ contains the geometrical dimensions of the spreading heat pulse above the heater. As a first approximation the geometry can be assumed as independent of the methanol concentration. Since the thermal conductivity of the methanol/water-mixture decreases by approx. $65 \%$ from pure water to pure methanol while the specific heat capacity only changes by approx. $40 \%$ and the excess heat capacity the difference between the actual heat capacity of the mixture and the idealized linear interpolation between the pure components [13] - is rather small with less than $8.3 \%$ [8] it was expected that the resulting time constant would show a linear dependence on the methanol concentration. However, the fit results show that the influenced region above the heater changes considerably with the methanol concentration resulting in a significant change of $\alpha$. Thus, a more complex mathematical model is necessary to describe the sensor principle.

The pre-exponential factors $a$ and $b$ show that the influence of the smaller time constant $\tau_{1}$ decreases while that of the larger time constant $\tau_{2}$ increases with increasing methanol concentration. The constant $c$ which reflects the theoretical steady state temperature of the heater shows a nearly linear dependence over a wide methanol concentration range with a low noise compared to the other parameters and can thus be used to determine the mixture ratio if the ambient temperature is not changed. The measured sensitivity using the constant $c$ is approx. $0.15 \mathrm{~K} / \mathrm{vol} \%$ with a precision of better than $\pm 0.2 \mathrm{~K}$ determined from the variation of the recorded temperature deviations from the linear correlation for all measurements. This results in an achievable accuracy of about $\pm 1.3 \mathrm{vol} \%$. This is slightly better than determining the concentration from the heater temperature increase after up to 2 seconds as shown in Fig. 6.

\section{Conclusion}

Microthermal sensors made of microstructured aluminum on a polyimide film with a very low thermal conductivity have been realized. Measurements have shown that mixtures of methanol and deionized water can be determined with an accuracy of about $2 \%$ within less than a second. In the range typically used for Direct Methanol Fuel Cells the increase of the heater temperature is almost linearly dependent on the mixture ratio.

Curve fitting seems to be an appropriate method for improving the determination of the methanol concentration, but also in achieving a better understanding of the sensor function principle. Here, the time constants seem to be depending strongly on the specific heat capacity of the methanol/water-mixture. However, the calculated time constants show a higher variation or noise requiring more heat pulses to determine the mixture ratio with sufficient accuracy. Only the fitted constant $c$ 
(eq. 1) has low noise and can be used to determine the mixture ratio. The accuracy achieved with this measurement principle is approx. $2.5 \%$, both for the fitted constant as when evaluating the temperature increase after 2 seconds. However, the influence of the ambient temperature on both measurement principles has to be evaluated and the computer performance required for fitting the measurement data must not be neglected, especially for low cost systems.

The developed sensor is also capable of detecting if the methanol/water-mixture is frozen (at below $-6^{\circ} \mathrm{C}$ for $10 \mathrm{wt} \%$ of methanol [14]) or if there is air in the sensor chamber which would both lead to malfunction in Direct Methanol Fuel Cells. That is due to the fact that the thermal conductivity of ice is much higher than that of the mixture which in turn is much higher than that of air, see Tab. 2.

Tab. 2: Comparison of thermal conductivity of ice, methanol/water-mixtures and air [7]

\begin{tabular}{|c|c|}
\hline Substance & $\begin{array}{c}\text { Thermal conductivity at } \\
20^{\circ} \mathrm{C} / \mathrm{W} / \mathrm{m} \cdot \mathrm{K}\end{array}$ \\
\hline Ice & $2.1^{(1)}$ \\
\hline Water & 0.59 \\
\hline $5 \mathrm{vol} \%$ methanol & 0.57 \\
\hline 10 vol\% methanol & 0.55 \\
\hline Methanol & 0.21 \\
\hline Air & $0.026^{(2)}$ \\
\hline
\end{tabular}

(1) at $0^{\circ} \mathrm{C}[10]$; $^{(2)}$ at $27^{\circ} \mathrm{C}[10]$

On the other hand, a major drawback of aluminum is its chemical instability. Long-term measurements have shown a continuous increase in the heater resistance because of corrosion. Therefore, a thin polyimide layer deposited via spin coating is currently being tested as a passivation layer on top of the sensor to encapsulate the aluminum structures. For this purpose Durimide $32 \mathrm{~A}$, a pre-imidized polyamide-imide (Fujifilm), was used to realize a coating thickness of approx. $2 \mu \mathrm{m}$ [15].

\section{Outlook}

Using an asymmetric sensor layout as shown in Fig. 2 allows making use of the varying distances of the temperature sensors from the heater to achieve multiple information and thus a more robust sensor principle. On the realized sensor chips the distances between the temperature sensors and the heater are varied between $0.25 \mathrm{~mm}$ and $2 \mathrm{~mm}$ with symmetric and asymmetric layouts. It will be tested weather a time-of-flight measurement of the spreading heat pulse can also be used to determine the mixture ratio.

Furthermore, measurements are currently done in stopped-flow mode. In the future, we will investigate the potential to simultaneously measure the flow rate with the sensor. For this purpose, the use of the temperature sensors arranged around the heater as a hot- or pulsedwire anemometer will be tested. This information could be used to compensate the concentration measurements in flow and also to further improve the control scheme of the DMFC system.

\section{Acknowledgement}

The authors thank the Federal Ministry of Education and Research (BMBF) for funding this research in the framework of the project InMischung (support code: 16SV5394) in the field of Microsystems Technology.

\section{References}

[1] M. Nölke, Entwicklung eines Direkt-MethanolBrennstoffzellensystems der Leistungsklasse kleiner $5 \mathrm{~kW}$, Forschungszentrum Jülich, Jülich, 2007, ISBN: 3-893-36481-1

[2] K. Scott, W. Taama, J. Cruickshank, Performance of a direct methanol fuel cell, Journal of Applied Electrochemistry 28 (3), 289297 (1998); doi: 10.1023/A:1003263632683

[3] A. A. Kulikovsky, The voltage-current curve of a direct methanol fuel cell: "exact" and fitting equations, Electrochemistry Communications 4, 939-946 (2002); doi: 10.1016/S1388-2481(02)00494-0

[4] A. Oedegaard, C. Hentschel, Characterisation of a portable DMFC stack and a methanol-feeding concept, Journal of Power Sources 158 (1), 177187 (2006); doi: 10.1016/j.jpowsour.2005.06.044

[5] X. Ren, P. Zelenay, S. Thomas, J. Davey, S. Gottesfeld, Recent advances in direct methanol fuel cells at Los Alamos National Laboratory, Journal of Power Sources 86 (1-2), 111-116 (2000); doi: 10.1016/S0378-7753(99)00407-3

[6] S. R. Narayanan, T. I. Valdez, Portable direct methanol fuel cell systems, Handbook of Fuel Cells, (2010); doi: 10.1002/9780470974001.f309094

[7] O. K. Bates, G. Hazzard, G. Palmer, Thermal Conductivity of Liquids, Industrial \& Engineering Chemistry Analytical Edition 10 (6), 314-318 (1938); doi: 10.1021/ac50122a006

[8] P. Westh, A. Hvidt, Heat capacity of aqueous solutions of monohydric alcohols at subzero temperatures, Biophysical Chemistry 46 (1), 27-35 (1993); doi: $10.1016 / 0301-4622(93) 87004-G$ 
[9] DuPont, DuPont Kapton HN, Kapton HN datasheet (2011)

[10] D. R. Ride, CRC Handbook of Chemistry and Physics, $88^{\text {th }}$ edition (2007-2008), CRC Press; ISBN: 978-0-8493-0488-0

[11] B. Schmitt, C. Kiefer, K. Kühn, A. Schütze, Flüssigkeitsanalyse mittels mikrothermischer Sensoren, 10. Dresdner Sensor-Symposium, 225-228 (2011); doi: 10.5162/10dss2011/12.7

[12] Methanex, Technical Information \& Safe Handling Guide for Methanol, Version 3.0 (2006)

[13] K. P. Shukla, A. A. Chialvo, J. M. Haile, Thermodynamic Excess Properties in Binary Fluid Mixtures, Industrial \& Engineering Chemistry Research 27(4), 664-671 (1988); doi: 10.1021/ie00076a023

[14] F. H. Conrad, E. F. Hill, E. A. Ballman, Freezing Points of the System Ethylene Glycol-MethanolWater, Industrial and Engineering Chemistry 32 (4), 542-543 (1940); doi: 10.1021/ie50364a023

[15] Fujifilm, Durimide 10/32 Pre-imidized Polyamideimide, Technical Product Information (2012) 